\title{
Photoresponsive Behavior of Orange and Red-Fluorescent Dyes in Nematic Liquid Crystals
}

\author{
Motoi Kinoshita ${ }^{1,2 *}$, Motoyuki Furukawa ${ }^{2}$, Saki Oka ${ }^{1}$, Yasuhiro Aoki ${ }^{1,2}$, Atsushi Shishido ${ }^{3,4}$ \\ ${ }^{1}$ Faculty of Engineering, Saitama Institute of Technology, \\ 1690 Fusaiji Fukaya Saitama 369-0293, Japan \\ ${ }^{2}$ Graduate School of Engineering, Saitama Institute of Technology, \\ 1690 Fusaiji Fukaya Saitama 369-0293, Japan \\ ${ }^{3}$ Laboratory for Chemistry and Life Science, Institute of Innovative Research, Tokyo Institute of Technology, \\ ${ }^{4}$ PRESTO, JST, Japan
}

Keywords: fluorescent dye, liquid crystal, photoinduced reorientation

\section{Introduction}

Control of molecular alignment in liquid-crystalline (LC) system has attracted increasing attention in recent years due to its application to alignment layer for LCDs, retardation films and refractive-index modulation devices [1]. LC system doped with dye molecules exhibits significantly enhanced photoinduced reorientation behavior compared to undoped LC system [2]. Although the reorientation behavior of the system has been interpreted as a phenomenon based on interaction between the dye molecules and the electric field of light, the effect of molecular structure of the dye on the photoinduced reorientation and details of the reorientational mechanism are not clarified.

Recently, we reported that green fluorescent coumarin dyes act as triggers for photoinduced reorientation of nematic LCs to reduce photothermal effect during photoirradiation at high-light intensity [3]. In this study, we explored photoresponsive behavior of nematic LCs containing orange and red-fluorescent dyes to create photoalignment systems with sensitivity in the long-wavelength region.

\section{Method}

\subsection{Sample Preparation}

Chemical structures of the guest dyes used in this study are shown in Fig. 1. Pyrromethene 580 was obtained from Exciton Inc. DCM1, Phenoxazone 660 and Solvent red 197 were obtained from TCI Ltd. E7 was obtained from
LCC Co. Ltd. used as a host LC without further purification and the guest dye was doped at a concentration of $0.1 \mathrm{~mol} \%$. Guest dyes and E7 were dissolved separately in THF and the solutions were mixed together, respectively. After the solvents were removed completely under vacuum, the LC mixtures were sandwiched between two parallel transparent glass substrates with a gap of $110 \mu \mathrm{m}$ by using double side tapes as a spacer. The inner surfaces of the two substrates were coated with $n$-octadecyl trimethoxy silane to obtain homeotropic alignment of LCs. The monodomain structure of the LC cell was confirmed by POM, and highly homeotropic alignment was observed. This cell was used as a sample for optical measurement.

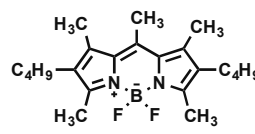

Pyrromethene $\mathbf{5 8 0}$
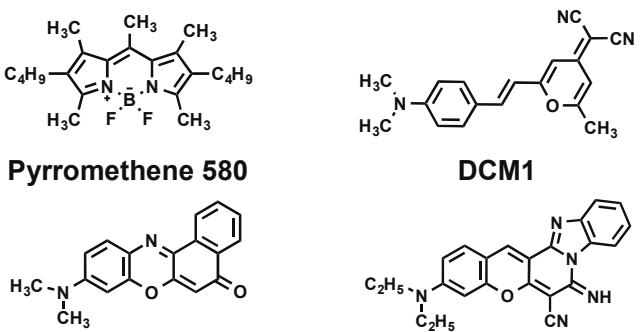

Phenoxazone 660

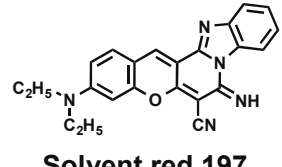

Fig. 1. Chemical structure of the dyes used in this study

\subsection{Optical Setup}

Photoresponsive behavior was evaluated by observation of self-diffraction patterns on a screen at room temperature. The optical setup is shown in Fig. 2. A linearly polarized Gaussian beam at 514 $\mathrm{nm}$ from an $\mathrm{Ar}^{+}$laser was focused normally onto the sample cell by a lens with a focal length of 200 
$\mathrm{mm}$ to induce reorientation of LC system. The polarization direction of the $\mathrm{Ar}^{+}$laser beam was maintained with a polarizer. The transmitted beam pattern created after the sample cell was observed on a screen behind the sample.

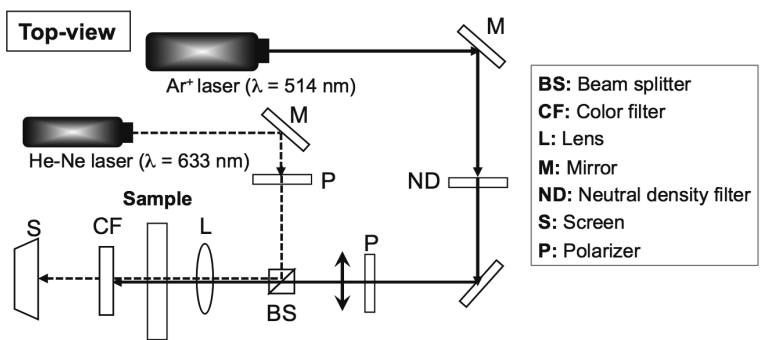

Fig. 2. Schematic representation of the experimental setup for the pump-probe measurement.

\section{Results and Discussion}

When the laser beam was incident on the sample cell above the threshold intensity, we observed several patterns on a screen formed by the transmitted light shown in Fig. 3. In the case of the sample cells doped with Pyrromethene 580 and DCM1, stable small rings were observed (Fig3a and $3 b)$. The images are clearly different from that of oligothiophene or coumarin derivative-doped sample in terms of the size and number of rings [3], which may attributed to thermal lens due to the energy of thermal deactivation from the excited dye [4]. The sample cell doped with Phenoxazone 660 did not show any diffraction pattern even at high irradiation intensity (Fig. 3c). For the sample cell doped with Solvent red 197, the clear diffraction pattern was observed (Fig. 3d). Appearance of the many diffraction rings indicates photoinduced reorientation of LCs, behaving in the same way as with oligothiophene and coumarin derivative.
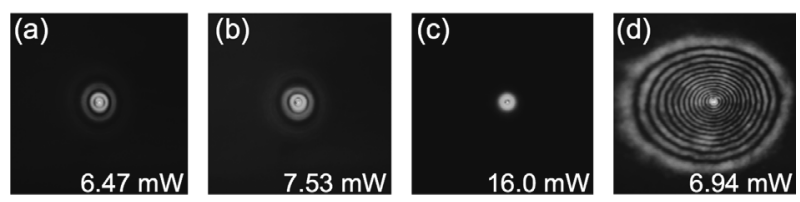

Fig. 3. Photographs formed on a screen observed in the sample cell doped with Pyromethene 580 (a), DCM1 (b), Phenoxazone 660 (c) and Solvent red 197 (d) at several irradiation intensities.

To investigate the alignment direction of LC system doped with solvent red 197, the polarization of the probe beam was maintained parallel or perpendicular to that of the pumping beam. We observed the clear diffraction pattern formed by the He-Ne probe beam with polarization parallel to that of the pumping beam, and no diffraction pattern formed by the probe beam with polarization perpendicular to that of the pumping beam. This phenomenon indicates that there is a gradient of the refractive index probed by parallel polarization, while no change in the refractive index is detected by perpendicular polarization. There is no large difference in the behavior of reorientation based on the oligothiophene [2] and the coumarin derivative [3]. Thus, Solvent red 197 was found to function as a dye for photoinduced reorientation of LC system. Further investigations about the dependence of the irradiated intensity and dye concentrations are now in progress.
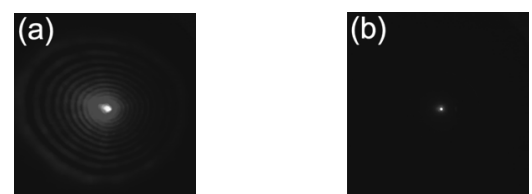

Fig. 4. Photographs of the diffraction patterns of the probe beam parallel (a), perpendicular (b) to the pumping beam.

\section{Conclusion}

Photoinduced reorientation behavior of orange and red-fluorescent dye-doped LCs were explored. Solvent red 197 was found to function as a dye for photoinduced alignment change of LC system.

\section{Acknowledgements}

This research was partially supported by Konica Minolta Science and Technology Foundation and the Ministry of Education, Science, Sports and Culture, Grant-in-Aid for Scientific Research (C) 25410210.

\section{References}

1. T. Ikeda, J. Mater. Chem., 13 (2003) 2037; A. Priimagi, C. Barrett, and A. Shishido, $J$. Mater. Chem. C, 2 (2014) 7155.

2. I. Janossy, A. D. Lloyd, and B. S. Wherrett, Mol. Cryst. Liq. Cryst., 179 (1990) 1; H. Zhang, S. Shiino, A. Shishido, A. Kanazawa, O. Tsutsumi, T. Shiono, and T. Ikeda, $A d v$. Mater., 12 (2000) 1336; M. Yaegashi, A. Shishido, T. Shiono, and T. Ikeda, Chem. Mater., 17 (2005) 4304; J. Wang, Y. Aihara, M. Kinoshita, and A. Shishido, Opt. Mater. Exp., 5 (2015) 538.

3. M. Kinoshita, Phys. Status Solidi (C), 9 (2012) 2637; M. Kinoshita and A. Shishido, Proc. SPIE, 8828 (2013) 88280A-1.

4. H. Zhang, S. Shiino, A. Kanazawa, O. Tsutsumi, T. Shiono, and T. Ikeda, J. Appl. Phys., 91 (2002) 5558. 